\title{
Avaliação do consumo e análise da rotulagem nutricional de alimentos com alto teor de ácidos graxos trans
}

\author{
Consumption and analysis of nutricional label of foods with high content of trans fatty acids
}

\author{
Juliana Ribeiro DIAS ${ }^{1}$, Édira Castello Branco de Andrade GONÇALVES ${ }^{1 \star}$
}

\begin{abstract}
Resumo
Nas últimas décadas, diversos estudos vêm sendo realizados visando avaliar os efeitos dos ácidos graxos trans sobre o organismo e identificar seu mecanismo de ação. Entretanto, somente a cerca de um ano, este item foi incluído na rotulagem nutricional obrigatória brasileira, permitindo ao consumidor controlar o consumo de ácidos graxos trans. Assim, o objetivo deste estudo foi avaliar a adequação de alguns alimentos com alto teor de ácidos graxos trans (biscoitos, sorvetes, chocolates e fast-food) frente à legislação pertinente e, ainda, o consumo diário por adultos e crianças observando a recomendação da OMS. A avaliação da rotulagem nutricional demonstrou que a maioria das amostras analisadas ainda não se adequou à nova legislação. Com base na análise dos questionários de consumo, identificou-se que 39,7\% dos adultos e 41,4\% das crianças consomem, diariamente, pelo menos um alimento com alto teor de ácidos graxos trans. Observou-se ainda, através do consumo estimado, que a ingestão parcial ou total destes produtos ultrapassa a recomendação diária para adultos (2 g) e crianças (1 ano-0,8 g e 10 anos-1,9 g). Assim, uma atuação efetiva dos órgãos de fiscalização e a promoção de ações educativas visando à menor utilização desses produtos na alimentação deveriam ser estimuladas.
\end{abstract}

Palavras-chave: ácidos graxos trans; consumo; rotulagem nutricional.

\begin{abstract}
Several studies have been carried over the last decades aiming at evaluating the effects of trans fatty acids on the organism and identifying their mechanism. However, only around one year ago it was included by law in the mandatory Brazilian nutritional food labeling. It now enables the consumer to control the consumption of trans fatty acids. The objective of this study was to evaluate the compliance of foods with high content of trans fatty acids (biscuits, ice cream, chocolate, and fast-food) with the legislation and the daily consumption recommendation by the OMS. The evaluation of Nutrition labeling indicated that some samples do not comply with the new legislation. Analysis of the questionnaires identified that $39.7 \%$ of adults and $41.4 \%$ of children consume daily at least one food with high content of trans fatty acids. The ingestion of these products exceeds the daily recommendation. Adequate fiscalization and healthy diet programs should be stimulated.

Keywords: trans fatty acids; consumption; nutrition labeling.
\end{abstract}

\section{Introdução}

Os Ácidos Graxos (AG) trans são um tipo específico de gordura proveniente do processo de hidrogenação natural (ocorrido no rúmen dos animais) ou industrial.

A aplicação da hidrogenação industrial objetiva principalmente a melhoria das características físicas (aparência, aroma, sabor, cor, textura) e sensoriais e conservação de um alimento (CHIARA et al., 2003).

Constituem fontes importantes de ácidos graxos trans na dieta os alimentos industrializados (bolos, biscoitos, chocolates, margarinas, sorvetes e produtos de fast-food) e, em menores quantidades, a carne, o leite e as gorduras de animais ruminantes. Sendo assim, o teor de ácidos graxos trans ingerido por uma população está diretamente relacionado com o padrão alimentar desta (BERTOLINO et al., 2006; VASCONCELOS COSTA et al., 2006).

Somente em 1990, através de um estudo realizado por Mensink e Katan (1990), a atenção de muitos pesquisadores foi despertada para a investigação dos efeitos adversos dos ácidos graxos trans. Segundo estudos, esses ácidos estão relacionados principalmente ao desenvolvimento de doenças cardiovasculares e alterações no crescimento e desenvolvimento fetal e infantil (BERTOLINO et al., 2006; CHIARA et al., 2003; SEMMA, 2002; TROISI; WILLET; WEISS, 1992).

Essa associação confirma a relevância da declaração do teor de ácidos graxos trans presente no alimento, prevista pela nova legislação da Agência Nacional de Vigilância Sanitária (ANVISA).

Segundo a RDC 360/03, devem ser incluídos na rotulagem nutricional obrigatória, até 31 de julho de 2006, o teor de ácidos graxos trans e a medida caseira da porção informada. Além disso, o valor energético diário deve ser reduzido para $2000 \mathrm{kcal}$ (BRASIL, 2000).

Entretanto, fica excluída a declaração de gorduras trans em percentual de valor diário (\%VD), já que ainda não há um

Recebido para publicação em 14/8/2007

Aceito para publicação em 17/2/2007 (002770)

${ }^{1}$ Laboratório de Análise de Alimentos, Departamento de Tecnologia de Alimentos, Escola de Nutrição, Universidade Federal do Estado do Rio de Janeiro - UNIRIO,

Av. Pasteur, 296, CEP 22290-240, Rio de Janeiro - RJ, Brasil, E-mail: ediracba@analisedealimentos.com.br

${ }^{*}$ A quem a correspondência deve ser enviada 
valor diário de ingestão preconizado pelos órgãos competentes. A recomendação é que seja consumido o mínimo possível e, segundo a OMS, não deve ultrapassar $1 \%$ do valor calórico da dieta. No Brasil, ainda não existem estudos populacionais para estimativa do consumo de ácidos graxos trans.

Diante do exposto, justifica-se a realização de estudo para levantamento do consumo de ácidos graxos trans e das concentrações destes em alimentos com alto teor (biscoitos, sorvetes, chocolates e produtos de fast-food), segundo a rotulagem nutricional.

A partir dos dados obtidos, busca-se estimar a quantidade de ácidos graxos trans ingerida provenientes de alimentos que contêm altos teores de ácidos graxos trans, verificando se há possibilidade de ultrapassar a recomendação da WHO referente a esses lipídios.

\section{Material e métodos}

\subsection{Rotulagem nutricional}

A análise da rotulagem nutricional baseou-se nos itens obrigatórios, segundo Regulamento Técnico Sobre Rotulagem Nutricional de Alimentos Embalados (RDC 360/03).

Entre os produtos industrializados (biscoitos Água \& Sal, "Cream Cracker" e recheados; chocolates e sorvetes), a análise baseou-se na rotulagem nutricional de 150 amostras de diferentes marcas, considerando dois lotes distintos. A coleta foi realizada em supermercados do Estado do Rio de Janeiro, no período de novembro/2006 a fevereiro/2007.

Para os produtos fast-food, foram avaliadas as informações nutricionais disponibilizadas por duas redes situadas no Estado do Rio de Janeiro, definidas como rede I e II (RI e RII), com 31 e 18 produtos, respectivamente. Tais produtos foram agrupados em hambúrguer; batata-frita; milk-shake; e sorvetes.

\subsection{Estudo descritivo de consumo}

A avaliação do consumo de alimentos com alto teor de ácidos graxos trans foi feita através da aplicação de um questionário de frequência alimentar entre 100 indivíduos frequentadores de um supermercado situado no município de Duque de Caxias-RJ, no período de dezembro/2006 a fevereiro/2007.

Esses indivíduos tinham opção de declarar se tais alimentos também eram ingeridos pelas crianças moradoras da mesma residência.

\section{Resultados e discussão}

\subsection{Adequação dos produtos industrializados e de fast-food à RDC 360/03}

Estudo realizado pela Agência Nacional de Vigilância Sanitária no $1^{\circ}$ semestre de 2005, avaliando 73 amostras, diz que veicular informação contrária à aprovada para rotulagem nutricional correspondeu a 51,4\% das infrações encontradas nas peças publicitárias de alimentos.
No presente estudo, apesar da análise da rotulagem nutricional ter sido iniciada quatro meses após o prazo máximo previsto pela Agencia Nacional de Vigilância Sanitária (ANVISA), observou-se, ao final do estudo, que mais da metade das amostras (55,3\%), principalmente os biscoitos recheados e os sorvetes, ainda não haviam sofrido as alterações obrigatórias.

A principal infração observada foi ausência da medida caseira equivalente à porção informada, omitida em 27,3\% das amostras, em sua maioria nos sorvetes $(67,7 \%)$.

Outro item inserido na nova rotulagem que também esteve ausente nas embalagens foi o teor de gorduras trans (22\%), principalmente nos sorvetes $(38,7 \%)$ e nos biscoitos recheados $(27,1 \%)$. A ausência dessa informação é extremamente relevante pela associação entre o consumo de elevados teores de ácidos graxos trans e maior risco de doenças cardiovasculares e pré-eclampsia (WILLIAMS et al., 1998; VASCONCELOS COSTA et al., 2006).

Estes resultados são semelhantes aos encontrados em uma pesquisa de mercado realizada no início de 2006 pelo Instituto Brasileiro de Defesa do Consumidor (IDEC), visando avaliar o cumprimento desta RDC em 370 produtos, quando foi observado que $37,6 \%$ da rotulagem desses produtos ainda não havia sido adequada à nova legislação quanto ao teor de gorduras trans.

Das amostras das redes de fast-food que tiveram a tabela nutricional avaliada, todas apresentavam as informações preconizadas pela RDC 360/03, inclusive quanto ao teor de gorduras trans, com exceção apenas à medida caseira equivalente à porção informada em sua tabela, que não foi encontrada nos alimentos da rede I.

\subsection{Teor de gorduras trans nos produtos analisados}

Os teores de ácidos graxos trans entre os biscoitos Água \& Sal e "Cream Cracker" são semelhantes. Entretanto, ambos os produtos declaram teores inferiores ao encontrado em outros estudos realizados no Brasil e no exterior (CHIARA; SCHIERI, 2001; ENIG et al., 1983; TAVELLA et al., 2000).

Na Figura 1, observam-se os teores de ácidos graxos trans mencionados na rotulagem nutricional das diferentes marcas de biscoitos Água \& Sal avaliados.

Na comparação entre as marcas, verifica-se que não há conformidade quanto ao teor de ácidos graxos trans, variando entre

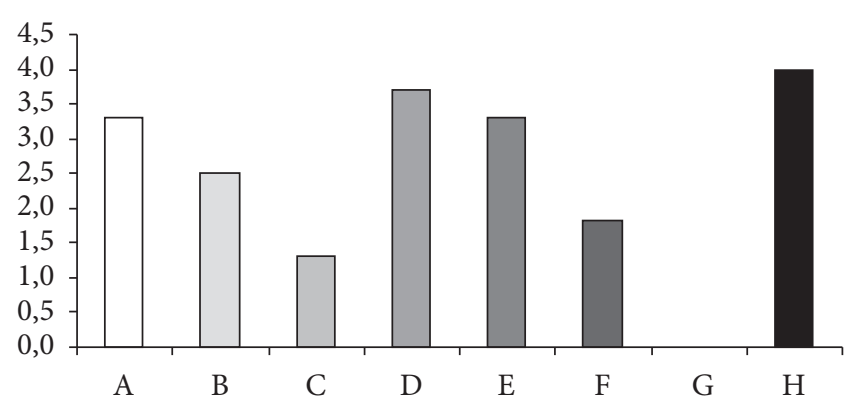

Figura 1. Teor de ácidos graxos trans $\left(\mathrm{g} .100 \mathrm{~g}^{-1}\right)$ mencionados nos rótulos de diferentes marcas de biscoitos Água \& Sal analisados $(\mathrm{N}=18)$. 
0 e 4,0\% (g.100 g $\mathrm{g}^{-1}$ ). Isto já causa uma dúvida ao consumidor quanto à qualidade do produto a ser consumido.

A não uniformidade entre as marcas dos biscoitos "Cream Cracker" (Figura 2) também é observada, apresentando teores de AG Trans entre 0 e 4,3 g.100 g-1.

A ausência de ácidos graxos trans na marca G, tanto para os biscoitos Água \& Sal quanto "Cream Cracker", deve-se à presença da gordura vegetal interesterificada.

O biscoito recheado foi o produto com maior teor, sendo superior a 5,0 g.100 g $\mathrm{g}^{-1}$ em 68,8\% das marcas (Figura 3). Esta informação é preocupante, uma vez que os biscoitos recheados são consumidos por grande parte da população infantil, e estudos vêm relacionando o consumo de grandes teores de ácidos graxos trans com alterações no crescimento e desenvolvimento fetal e infantil (CRAWFORD, 2000).

É importante ressaltar que grandes discrepâncias são encontradas entre marcas de uma mesma empresa, já que enquanto o biscoito B não possui teores significativos de AG Trans o biscoito A, da mesma empresa, apresenta cerca de 6,0\% (g.100 g $\left.\mathrm{g}^{-1}\right)$.

Quanto às amostras de chocolate, verificou-se que 66,7\% das marcas apresentaram teores não significativos de ácidos graxos trans. Somente os produtos de uma empresa (marcas I, $\mathrm{L}, \mathrm{M}$ e N) declaram conter ácidos graxos trans em quantidades significativas, variando entre 0,8 e $16,7 \mathrm{~g} .100 \mathrm{~g}^{-1}$.

Observando as diferentes marcas de sorvete, os produtos das marcas $\mathrm{H}$ e L foram os únicos a apresentar teores significativos de ácidos graxos trans, em torno de 2,0 g\% ( $\left..100 \mathrm{~g}^{-1}\right)$.

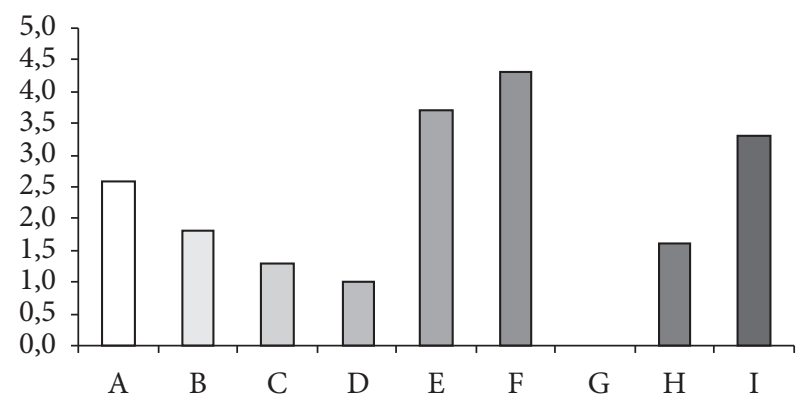

Figura 2. Teor de ácidos graxos trans (g. $100 \mathrm{~g}^{-1}$ ) mencionados nos rótulos de diferentes marcas de biscoitos "Cream Cracker" $(\mathrm{N}=19)$.

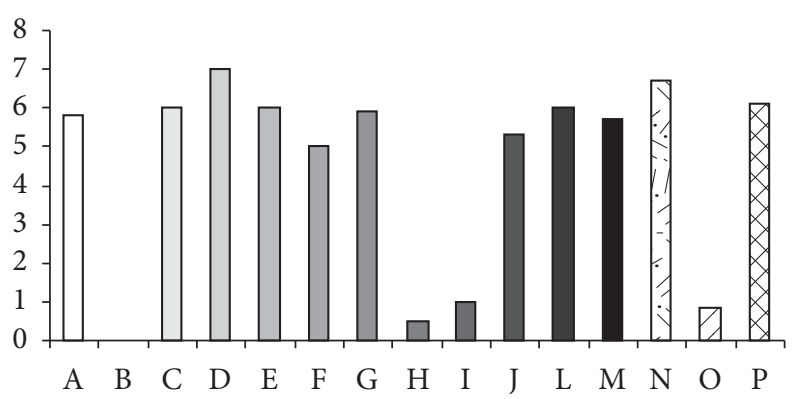

Figura 3. Teor de ácidos graxos trans (g.100 g $\mathrm{g}^{-1}$ ) mencionados nos rótulos de diferentes marcas de biscoitos recheados $(n=48)$.
Dessa forma, a escolha por determinada marca teria papel fundamental no teor de ácidos graxos trans ingeridos por um indivíduo.

A Figura 4 apresenta a distribuição do teor de gorduras trans nos produtos comercializados pelas duas redes de fast-food baseado nas informações nutricionais oferecidas pelas tabelas nutricionais destas empresas.

Quanto ao teor de gorduras trans, somente o hambúrguer (RII) e a batata-frita (RI) declaram-se livre de ácidos graxos trans. Os maiores teores são declarados pelo milk-shake da RII.

Em estudo realizado por Chiara et al. (2003), visando dosar o teor de AG trans em alguns alimentos consumidos no Rio de Janeiro, elevados teores de ácidos graxos trans foram encontrados na batata-frita das redes de fast-food. No presente estudo, segundo a tabela nutricional, o produto da RII contém metade do teor de ácidos graxos trans observado pelos autores e o produto da RI é livre de gorduras trans, justificado pelo rígido controle com a filtragem, troca constante do óleo usado nas frituras e a combinação do óleo de palma e algodão nos processos de fritura adotados, conforme informação da empresa.

\subsection{Avaliação do consumo de Ácidos Graxos Trans}

O consumo de AG Trans foi avaliado através da aplicação de questionário com 100 indivíduos aleatoriamente distribuídos em: $67,2 \%$ mulheres e $32,8 \%$ homens.

A frequência do consumo de alimentos com alto teor de ácidos graxos trans, pela população adulta e infantil, encontra-se nas Figuras 5 e 6, respectivamente.

Entre a população adulta, 39,7\% dos entrevistados consumiam diariamente pelo menos um alimento com alto teor de ácido graxo trans e somente $8,8 \%$ declararam nunca consumi-los.

Verifica-se que os itens mais indicados no consumo mensal entre os adultos são os biscoitos "Cream Cracker" (63,1\%), os biscoitos recheados (58,7\%) e os chocolates (57,3\%). No entanto, somente o biscoito "Cream Cracker" apresenta consumo diário elevado, enquanto os biscoitos recheados e os chocolates são consumidos uma vez na semana ou eventualmente, pela maioria.

Os produtos provenientes das redes de fast-food possuem elevado consumo mensal, sendo consumidos preferencialmente uma vez na semana.

Figura 4. Teor de gorduras trans mencionado nas tabelas nutricionais de produtos de redes fast-food (g/por porção) $(\mathrm{N}=49)$.

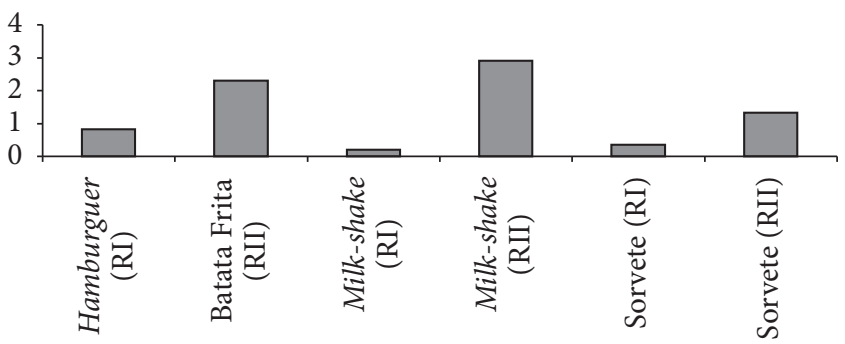




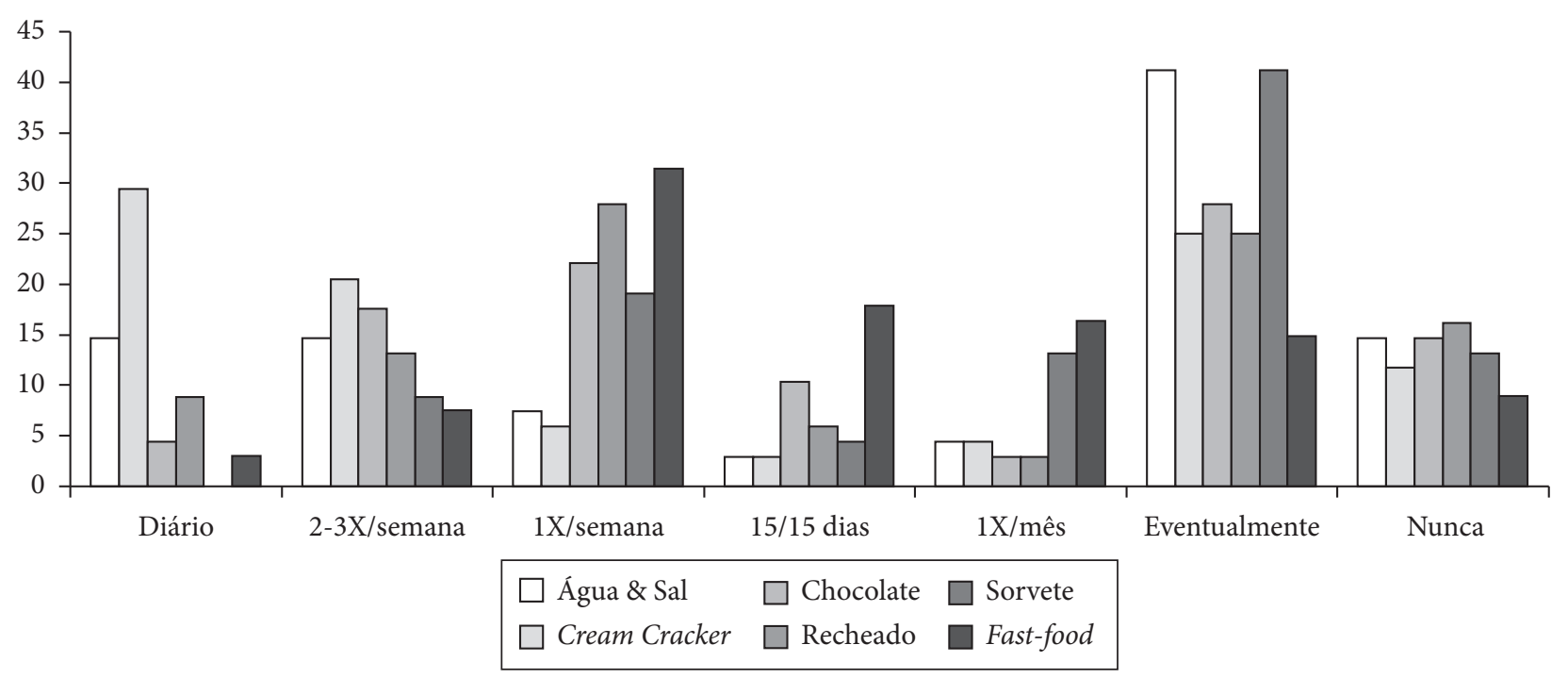

Figura 5. Frequência (\%) de consumo de alimentos com alto teor de ácidos graxos trans pela população adulta.

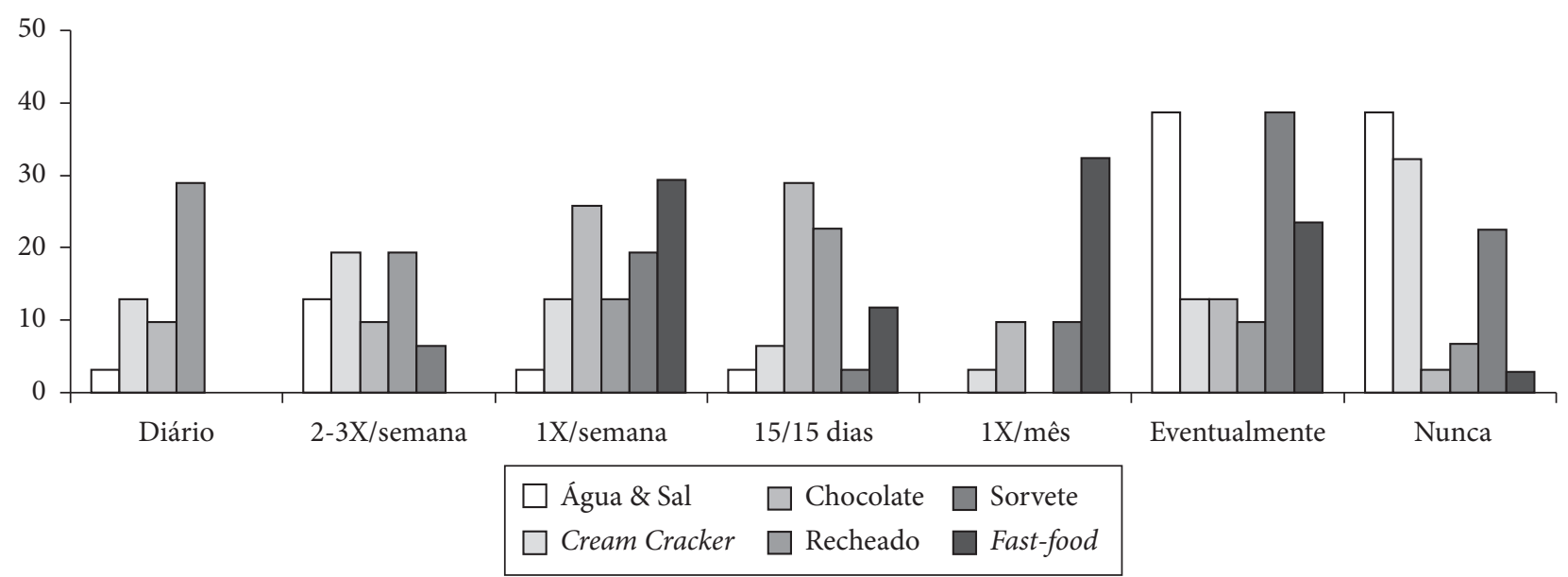

Figura 6. Frequência (\%) de consumo de alimentos com alto teor de ácidos graxos trans pela população infantil.

A população infantil analisada foi composta de 46 crianças, com idade entre 1 e 10 anos, das quais $41,4 \%$ consumiam pelo menos um alimento com alto teor de ácidos graxos trans.

Segundo Aued-Pimentel (2003), os biscoitos são largamente consumidos por crianças e adolescentes. Dentre a população infantil, observa-se um elevado consumo de biscoitos recheados, ricos em gorduras trans, sendo este, principalmente, diário. Entretanto os biscoitos Água \& Sal e "Cream Cracker" nunca são consumidos por cerca de $45 \%$ da população infantil.

Esta população estaria, portanto, mais suscetível a alterações fisiológicas importantes no que se refere ao crescimento e desenvolvimento infantil. Segundo estudos realizados, os isômeros trans atuam sobre a saúde da criança, bloqueando e inibindo a biossíntese dos ácidos graxos poli-insaturados de cadeia longa, que estão envolvidos com a função psicomotora. (BOOYENS; MERWE, 1992; KOLETZKO; MULLER, 1990)

Os chocolates consumidos eventualmente pela maior parte da população adulta apresentam elevado consumo semanal e quinzenal entre as crianças, além de maior consumo diário.

Em estudo realizado por Caroba (2002) entre outubro e novembro de 2000, visando avaliar o consumo alimentar de estudantes da rede pública de ensino, observou-se que $17,8 \%$ dos escolares que se alimentam fora de seu domicílio uma vez na semana utilizam o fast-food para realização de suas refeições.

Assim como a população adulta, os produtos de fast-food apresentam um expressivo consumo semanal $(29,4 \%)$ e mensal $(73,6 \%)$. Entretanto, observa-se que um maior número de crian- 
ças, em relação à população adulta, consome o produto eventualmente e que não há consumo diário ou 2 a 3 vezes/semana significativo entre as crianças.

\subsection{Estimativa de ingestão diária de AG Trans}

Considerando as informações obtidas nos rótulos dos produtos analisados e o questionário aplicado, foi estimada a ingestão de ácidos graxos trans para um indivíduo adulto (Tabela 1), visando observar se o consumo destes poderia ultrapassar a recomendação da Organização Mundial de Saúde (OMS, 2003). A OMS estipula que o consumo diário de ácidos graxos trans deve ser igual ou inferior a $1 \%$ do valor calórico total, ficando em torno de 2,0 g/dia para um indivíduo adulto que consome diariamente $2000 \mathrm{kcal}$.

Observa-se que o consumo total ou parcial acima estimado é capaz de ultrapassar facilmente a ingestão diária recomendada pela OMS, já que o consumo isolado destes produtos contribui com no mínimo $65 \%$ desta recomendação.

Este dado é preocupante na medida em que outros alimentos não pesquisados neste trabalho contêm essa fração lipídica e pela associação deste tipo de ácido graxo com o desenvolvimento de doenças cardiovasculares.

Com base na mesma recomendação da OMS (2003), uma criança entre 1 e 10 anos poderia consumir de 0,8 a 1,85 g/dia de AG Trans. Assim, a tabela 2 apresenta uma estimativa de consumo de ácidos graxos trans a partir da análise dos rótulos dos produtos analisados e do questionário aplicado.

Tabela 1. Consumo diário estimado de produtos com alto teor de AG Trans por um indivíduo adulto.

\begin{tabular}{|c|c|c|c|c|}
\hline \multirow[t]{2}{*}{ Refeição } & \multirow[t]{2}{*}{ Alimento } & \multicolumn{2}{|c|}{ Quantidade } & \multirow{2}{*}{$\begin{array}{c}\text { Teor de AG } \\
\text { Trans }(\mathrm{g})\end{array}$} \\
\hline & & Porção & g ou $\mathrm{mL}$ & \\
\hline Desjejum & $\begin{array}{c}\text { Biscoito "Cream } \\
\text { Cracker" } \\
\text { (Marca F) }\end{array}$ & 6 unidades & 30 & 1,3 \\
\hline \multirow[t]{3}{*}{ Almoço } & $\begin{array}{c}\text { Hambúrguer } \\
\text { (RII) }\end{array}$ & 1 unidade & - & 0 \\
\hline & $\begin{array}{l}\text { Batata- frita } \\
\text { (RII) }\end{array}$ & 1 pacote médio & 100 & 2,3 \\
\hline & $\begin{array}{l}\text { Milk-shake } \\
\text { (RII) }\end{array}$ & 1 copo médio & 300 & 2,9 \\
\hline Lanche & $\begin{array}{l}\text { Chocolate } \\
\text { (Marca E) }\end{array}$ & $\begin{array}{l}4 \text { unidades } \\
\text { pequenas }\end{array}$ & 24 & 4,0 \\
\hline Jantar & $\begin{array}{l}\text { Sorvete } \\
\text { (Marca A) }\end{array}$ & 1 bola & 60 & 1,3 \\
\hline Total & & & & 11,8 \\
\hline
\end{tabular}

Tabela 2. Consumo diário estimado de produtos com alto teor de AG Trans por uma criança entre 1 e 10 anos.

\begin{tabular}{lcccc}
\hline Refeição & Alimento & \multicolumn{2}{c}{ Quantidade } & \multirow{2}{*}{$\begin{array}{c}\text { Teor de AG } \\
\text { Trans }(\mathrm{g})\end{array}$} \\
\cline { 3 - 4 } Colação & $\begin{array}{c}\text { Biscoito recheado } \\
\text { (Marca L) }\end{array}$ & 6 unidades & 90 & 6,0 \\
Lanche & $\begin{array}{c}\text { Chocolate } \\
\text { (Marca E) }\end{array}$ & $\begin{array}{c}4 \text { unidades } \\
\text { pequenas }\end{array}$ & 24 & 4,0 \\
Total & & & & 10,0 \\
\hline
\end{tabular}

Na população infantil, observa-se também um consumo elevado de ácidos graxos trans, ultrapassando de 5 a 12 vezes a recomendação, e que a ingestão isolada de um dos produtos já ultrapassaria o consumo diário preconizado.

A situação toma maior importância uma vez que estamos utilizando apenas dois alimentos com alto teor de AG Trans e que outros podem compor a alimentação infantil.

\section{Conclusões}

Através do estudo realizado, observou-se que a maior parte dos produtos analisados (55,3\%) ainda não se adequou à norma prevista pela legislação, principalmente pela ausência da medida caseira da porção informada e o teor de ácidos graxos trans.

Com o auxílio dos rótulos dos produtos estudados, pode-se verificar que constituíram a principal fonte de gorduras trans os biscoitos recheados e alguns produtos provenientes de redes fast-food, principalmente da RII.

$\mathrm{Na}$ análise do consumo através da aplicação do QFA, observa-se que o consumo de biscoitos "Cream Cracker" é bastante difundido entre a população adulta e infantil e dos biscoitos recheados somente entre a população infantil. Elevado consumo semanal de produtos provenientes das redes de fast-food também é encontrado em ambas as populações. Devem, portanto, ser promovidas ações educativas que visem à redução do consumo de alimentos com alto teor de ácidos graxos trans e à promoção de uma alimentação mais saudável.

Ao estimar a contribuição para IDA dos alimentos com alto teor de ácidos graxos trans estudados, verificou-se que a ingestão destes supera facilmente a recomendação diária da OMS (2003), dado este preocupante na medida em que efeitos metabólicos diversos vêm sendo associados ao consumo elevado destes ácidos graxos.

Diante do exposto, constata-se que é importante que os órgãos de fiscalização tenham uma atuação mais ativa, punindo as empresas que ainda não de adequaram à RDC360/03. É necessária a determinação dos teores de ácidos graxos trans nos produtos para verificar a veracidade das informações contidas nos rótulos.

\section{Referências bibliográficas}

AUED-PIMENTEL, $S$ et al. Ácidos graxos saturados versus ácidos graxos trans em biscoitos. Rev. Instituto Adolfo Lutz, v. 62, n. 2, p. 131-37, 2003.

BERTOLINO, C. n. et al. Influência do consumo de ácidos graxos trans no perfil de lipídeos séricos em nipo-brasileiros de Bauru, São Paulo, Brasil. Caderno de Saúde Pública, v. 22, n. 2, p. 357-364, 2006.

BOOYENS, J.; MERWE, V. C. F. Margarines and coronary artery disease. Medicals Hypotheses, v. 37, n. 4, p. 241-244, 1992.

BRASIL. Resolução RDC n.360, de 23 de dezembro de 2003. Regulamento Técnico sobre Rotulagem Nutricional de Alimentos Embalados. Diário Oficial da República Federativa do Brasil, Brasília, DF, de 22 de dezembro 2000.

CAROBA, D. C. R. A Escola e o consumo alimentar de adolescentes matriculados na rede pública de ensino. São Paulo, 2002. 162p. Dissertação (Mestrado) - Escola Superior de Agricultura Luiz de Queiroz. 
CHIARA, V. L. et al. Teores de ácidos graxos trans de alguns alimentos consumidos no Rio de Janeiro. Revista de Nutrição, v. 16, n. 2, p. 227-233, 2003.

Chiara, V. L.; Sichieri, R. Food consumption of adolescents - A simplified questionnaire for evaluating cardiovascular risk. Arquivo Brasileiro de Cardiologia, v. 77, n. 4, p. 337-341, 2001.

CRAWFORD, M. A. Placental delivery of arachidonic and docosahexaenoic acids: implications for the lipid nutrition of preterm infants 1, 2, 3. American Journal of Clinical Nutrition, v. 71, n. 1, p. 275-284, 2000.

ENIG, M. G. et al. Fatty acid composition of the fat in selected food items with emphasis on trans components. Journal American Oil Chemical Society, v. 60, n. 10, p. 1788-1795, 1983.

KOLETZKO, B.; MÜLLER, J. Cis- and trans- fatty acids in plasma lipids of newborn infants and their mothers. Biology of the Neonate, v. 57, n. $3 / 4$, p. $172-178,1990$.
SEMMA, M. Trans fatty acids: properties, benefits and risks. Journal Health Science, v. 48, p. 7-13, 2002.

TAVELLA, M. et al. Trans fatty acid content of a selection of foods in Argentina. Food Chemistry, v. 69, n. 2, p. 209-213, 2000.

Troisi, R.; Willet, W. E.; Weiss, S. Trans-fatty acid intake in relation to lipid concentrations in adult men. American Journal of Clinical Nutrition, v. 56, n. 6, p. 1019-1024, 1992.

WILLIAMS, M. A. et al. Risk of preeclampsia in relation to elaidic acids(trans fatty acid) in maternal erythrocytes. Ginecologic and Obstetric Investigation, v. 46, n. 2, p. 84-87, 1998.

VASCONCELOS COSTA, A. G. et al. Ácidos Graxos Trans: Alimentos e Efeitos na Saúde. ALAN, v. 56, n. 1, p. 12-21, 2006. 